\title{
EL AGUA COMO ELEMENTO DE REFLEXIÓN ÉTICO-POLÍTICA EN EL NUEVO PARADIGMA DE LA GOBERNABILIDAD MIGRATORIA
}

\author{
WATER AS AN ELEMENT OF ETHICAL-POLITICAL REFLECTION IN \\ THE NEW PARADIGM OF MIGRATORY GOVERNANCE
}

\author{
María Francisca Zaragoza Martí \\ Universidad de Alicante, Alicante. España/Spain \\ maria.zaragoza@ua.es
}

Recibido/Received: 09/05/2017

Modificado/Modified: 04/07/2017

Aceptado/Accepted: 10/10/2017

\section{RESUMEN}

La gobernanza mundial se encuentra en una situación de crisis como consecuencia del mundo global y multirrelacional en el que nos encontramos, que si bien contribuye a las acciones conjuntas de mejora también genera mayores situaciones de desigualdad y discriminación. Es por ello que los Derechos Humanos se sitúan en el centro de los debates políticos y sociales actuales, pues es necesaria una revisión y adecuación de la regulación y la protección de los elementos más básicos de los mismos para hacerlos extensibles a la universalidad de los individuos, especialmente exigible a grupos de personas, como los migrantes, que se encuentran en el limbo jurídico de ningún Estado. Uno de estos elementos primordiales a través de los que se satisface la vida misma es el adecuado acceso al agua y al saneamiento, como basamento del resto de Derechos Humanos.

\section{PALABRAS CLAVE}

Gobernabilidad; Derechos Humanos; agua; migración; desarrollo.

\section{SUMARIO}

1. Introducción. 2. Los nuevos paradigmas jurídico-políticos que conectan migración y derecho universal al agua. 3. El agua como elemento de reflexión. 4. A modo de conclusión. Bibliografía.

\begin{abstract}
Global governance is in a crisis situation as a result of the global and multi-relational world in which we find ourselves, which, while contributing to joint actions for improvement, also creates greater situations of inequality and discrimination. That is why human rights are at the center of current political and social debates, as it is necessary to review and adapt the regulation and protection of the most basic elements of them to extend them to the universality of individuals. Especially for groups of people, such as migrants, who are in the legal limbo of any State. One of these primordial elements through which life itself is satisfied is adequate access to water and sanitation, as a basis for the rest of Human Rights.
\end{abstract}

\section{KEYWORDS}

Governance, Human Rights, water; migration; development. 


\section{CONTENTS}

1. Introduction. 2. The new legal-political paradigms that connect migration and universal right to water. 3. Water as an element of reflection. 4. By way of conclusion. References.

\section{INTRODUCCIÓN}

Desde los catastróficos acontecimientos producidos el 11-S, la Humanidad ha cambiado su forma de interrelacionarse en los diferentes sectores que conforman la pacífica convivencia de los seres humanos dentro y fuera de un Estado, de forma que las relaciones transfronterizas entre los Estados no son garantía suficiente para el adecuado respeto de los derechos inherentes al ser humano, independientemente de su origen, raza, etnia, sexo o religión. Ello ha generado una recesión o retroceso hacia los esquemas más clásicos de catalogación de los derechos, lo que ocasiona que el ejercicio de determinados derechos básicos para el desarrollo humano y social del hombre se someta a criterios de poder no accesibles a la generalidad de los seres humanos. Como consecuencia, no sólo grupos de personas determinadas, como los inmigrantes, las mujeres y los niños, sino también derechos fundamentales, como el derecho al agua, a la alimentación y a la salud, se quedan al margen del sistema de garantías de los Estados.

Ya decía Rousseau (2007: 37-38) que "el hombre ha nacido libre y, sin embargo, vive en todas partes entre cadenas. Tal cual se cree el amo de los demás, cuando, en verdad, no deja de ser tan esclavo como ellos"; claro ejemplo de lo que en la actualidad miles de personas viven (2.4000 millones de personas carecen de condiciones sanitarias dignas-Organización Mundial de la Salud y Unicef, 2014: 32), tanto en sus Estados como en aquellos otros a los que migran, por diversas causas, ya que sus derechos son habitualmente violados, a pesar de la obligación que sobre todo Estado recae de velar por el bienestar de sus ciudadanos, de garantizar y mantener las condiciones necesarias para que toda persona goce de sus derechos en toda su extensión.

Como consecuencia de ello, la geopolítica mundial se va a ver enfrentada a importantes cambios negativos si no es capaz de escuchar las nuevas demandas sociales. Las futuras guerras van a ser las guerras por los recursos naturales, en cuyo centro se situará el elemento vital para la supervivencia humana, como es el agua. Los efectos más devastadores de ello se podrán sentir en los países en vías desarrollo, especialmente sobre los grupos de personas más desfavorecidas, como lo son las mujeres, los niños y las personas migrantes, obligadas a no tener un Estado propio y a fluir de una frontera a otra buscando garantizar y salvaguardar su propio bienestar, ámbito humano que debería ser básico y fundamental (recordemos que el Estado tiene una de las tareas más trascendentales, cual es la de proteger los Derechos Humanos de todo individuo, obligándose a mantener las condiciones necesarias para que toda persona goce de sus derechos, del acceso a la paz, a la justicia, a la libertad y al bienestar común. Landero, 2015: 140).

\section{LOS NUEVOS PARADIMAS JURÍDICO-POLÍTICOS QUE CONECTAN MIGRACIÓN Y DERECHO UNIVERSAL AL AGUA}

La necesidad de originar un nuevo paradigma es igualmente aplicable tanto a la política migratoria internacional como a la exigencia del reconocimiento del Derecho Humano al 
agua, pues desde sus orígenes los flujos migratorios tienen como objetivo fundamental conseguir alcanzar una status cualitativamente mejor en el bienestar de las personas que migran. Y aunque existen diferentes causas que nos impulsan a migrar (económicas, demográficas, sociales, religiosas, bélicas...) y, por tanto, también diferentes categorías de migraciones (migración interna, externa, internacional, temporal, permanente, voluntaria, forzosa, brain drain...), los flujos migratorios son consustanciales al desarrollo de la sociedad humana en cuanto a que su propia dinámica es generadora de desequilibrios que desembocan en la polarización económica y social de la población (Cortizo, 1993: 22). De ahí que la misma se vea en la necesidad de seguir buscando hasta alcanzar un equilibrio de bienestar que le permita disfrutar de la vida en condiciones óptimas (vivir y no sólo sobrevivir, para lo que necesitan el elemento más básico del que nos formamos, el agua) y en cuyo epicentro, sin duda, se encuentra la garantía y salvaguarda de los derechos más básicos y necesarios para el hombre, cuyo ejercicio debe ser garantizado, protegido y promovido por los Estados con carácter universal sin distinción alguna.

\subsection{Los Derechos Humanos como enclave de la gobernabilidad migratoria}

Ambos elementos van unidos, dado que el reconocimiento de la dignidad humana como elemento transversal de los Derechos Humanos se sitúa más allá de cualquier requisito o exigencia política $\mathrm{y}$, por tanto, igualmente reconocible a las personas inmigrantes, más cuando migración y desarrollo social de un Estado suelen ir de la mano. De ahí la exigencia de establecer unas políticas adecuadas para la efectiva regulación de la migración y la necesidad de no seguir manteniendo las actuales regulaciones unilaterales que los Estados aplican, dado que ello ha generado un fallo en la gobernanza mundial y un retroceso en los marcos de actuación vinculantes de Naciones Unidas (Comisión Interamericana de Derechos Humanos, 2013). Debemos dar un giro hacia la denominada gobernabilidad migratoria, implementando políticas que coloquen a los Derechos Humamos de los migrantes como uno de los principales fundamentos de cambio, pues una aproximación que relacione ambas categorías junto con la noción de desarrollo, permitiría comprender determinadas prácticas estatales y no estatales dirigidas a la regulación de las fronteras internacionales (Domenech, 2013: 1-2).

Los Estados y los Organismos Internacionales han adquirido como propios los efectos que la era de la globalización está generando, de forma que ya no es posible tratar los diferentes asuntos de un Estado de forma unilateral y al margen del resto de los agentes políticosociales, sino a través de la implementación conjunta y universal de estrategias que garanticen una gobernabilidad soberana sustentada sobre un nuevo paradigma, un cambio de enfoque que fluya de una visión basada en la seguridad de las fronteras y en la salvaguarda del régimen interior del Estado a una posición avanzada y propia de los Estados sociales y democráticos modernos en los que los Derechos Humanos de las personas que lo conforman se constituyan como la piedra angular sobre la que pendule dicho sistema. Ya lo decía Mezzadra (2012: 169) al exclamar que el poder se está reconfigurando en un contexto en el que la lógica de la soberanía se entrelaza con la lógica de la gobernabilidad neoliberal, con una gobernanza que se presenta como un sencillo proceso de persuasión sin coerción.

Para garantizar la adecuada funcionalidad de este nuevo paradigma migratorio erigido sobre las personas como seres humanos y no como simples inputs económicos es necesario, a la par, desarrollar un sistema universal y transversal de garantía de los derechos sobre los que se desenvuelven las sociedades y las personas que las forman, como salvaguarda del adecuado funcionamiento de sistema migratorio, porque la protección de los Derechos Humanos resulta relevante en la medida en que la violación de los mismos provocaría 'flujos 
migratorios desordenados y no deseados', al mismo tiempo que el carácter 'desordenado y no deseado' de éstos supondría un riesgo mayor de violación de los Derechos Humanos (Ghosh, 2008:50).

Se necesita un enfoque multidimensional con soluciones sostenibles en el tiempo, flexibles y adaptables a las situaciones cambiantes del mundo, pues las costumbres y la cultura son conceptos de vida capaces de adaptarse y evolucionar para estar en sintonía con los Derechos Humanos (DESC, 2016: 6). Como bien mencionaba Loperena Rota (1999) los Derechos Humanos se han convertido en el parámetro clave del desarrollo civilizatorio, con un elenco de principios ético-políticos que debidamente juridificados se convierten en el basamento de cualquier sistema jurídico.

\subsection{Naciones Unidas como herramienta de acción del nuevo paradigma}

Esta afirmación nos permite reclamar la necesidad de cumplir y dar efectividad al enunciado marco que la Declaración Universal de Derechos Humanos (DUDH) realiza en su artículo 3: Todo individuo tiene derecho a la vida, a la libertad y a la seguridad de su persona, concretado en el contenido que la misma declaración realiza en su artículo 25 al mencionar que: Toda persona tiene derecho a un nivel de vida adecuado que le asegure, así como a su familia, la salud y el bienestar y, en especial, la alimentación, el vestido, la vivienda, la asistencia médica y los servicios sociales necesarios. Esta es una de la diversas razones por las que las personas migran, buscando alcanzar lo aquí mencionado, no sólo para sí sino también para su familia y ello es lo que hay que seguir respetando en el país de destino, atendiendo a las necesidades reales de las personas, dando efectividad a sus derechos, caminando hacia el nuevo paradigma de la gobernabilidad migratoria.

Así lo entiende el grupo ad hoc sobre Pobreza y Derechos Humanos de la Comisión de Derecho Humanos de Naciones Unidas que, una vez más, ha reiterado la indisoluble relación entre el derecho a la vida y la satisfacción de las necesidades humanas básicas (Doc. E/CN.4/Sub.2/2002/15: 3, 15 y 17); específicamente, el nivel más esencial del derecho a la vida es la manutención del nivel biológico de los seres humanos y, en esa medida, el derecho a la vida se descompone en cuatro derechos esenciales: el derecho a la alimentación adecuada, el derecho a contar con agua potable, el derecho a la vivienda y el derecho a la salud. Por tanto, la ausencia de alguno o varios de estos elementos constituiría una violación de la dignidad de los seres humanos, al igual y al mismo nivel de violencia como cuando se tortura, es decir, que el hambre y la falta de agua potable suponen una violencia extrema sobre los individuos (Ausín, 2013).

Esta conexión indisoluble y natural entre la realización del derecho a la vida y la satisfacción de las necesidades básicas sin las cuales la vida no tendría sentido, necesariamente debe ser tenida en cuenta por los Estados a la hora de implementar sus diversas políticas; más en concreto y teniendo en cuenta la era global en la que vivimos donde todas las partes del mundo están interconectadas y los flujos de personas son constantes, los Estados deben ser conscientes no sólo de cumplir con las obligaciones que como ente garante los mismos les asignan, sino que deben darse cuenta que con el reconocimiento, la protección y la regulación adecuada y universal de los bienes y derechos básicos de la Humanidad, mejoran, a la par, la calidad de la vida de las personas que van y viene de sus territorios, garantizando un mejor y mayor desarrollo social, promoviendo valores de integración e igualdad entre su población, con independencia de su origen, pues si los ciudadanos se sienten seguros y satisfechos en sus derechos protegerán y respetarán el Estado en el que viven. 


\subsection{La exigibilidad del carácter humano del recurso vital básico como consecuencia de los nuevos reclamos sociales}

Desafortunadamente y al igual que ocurre con la ausencia de una adecuada gobernabilidad migratoria por entender a las personas migrantes no como lo que son sino como costes o beneficios económicos, el agua (al igual que sucede con el acceso a la tierra y a los alimentos), es tratada por los organismos internacionales como un bien comercializable, sujeto a la especulación del mercado, lo que genera que su distribución no atienda a razones de equidad, sino de economía, ya que irán destinadas en función de la capacidad de pagar de cada destinatario. Ello impide revertir las consecuencias nocivas de la hambruna y la pobreza que sufre el mundo, especialmente en los países en vías de desarrollo y que igualmente afecta a las persona que migran, dado que sin un reconocimiento universal del Derecho Humano a acceder al agua y al saneamiento (evitando las altas tasas de mortalidad que genera no tener acceso al saneamiento, al agua limpia y pura), se contribuye de manera crónica a acentuar las diferencias entre norte y sur y a sumergir en un círculo vicioso de enfermedad y pobreza a millones de personas (el $21 \%$ de las muertes en niños menores de 5 años se debe a las constates diarreas producidas por no tener acceso a agua en condiciones adecuadas - OMS y UNICEF, 2014). Como nos recuerda el PNUD (2006) más allá del desgaste y el sufrimiento humanos, el déficit mundial del agua y saneamiento está socavando la prosperidad y retardando el crecimiento económico.

En atención a esta situación, no es de extrañar que nos encontremos, cada vez con mayor frecuencia, reclamos sociales sobre una nueva estrategia entorno al recurso hídrico y al reconocimiento del agua como un Derecho Humano, con nuevos enfoques más sociales y solidarios sobre el agua y la gestión, sin tener que vulnerar los derechos que son propios del ser humano. En este contexto, encontramos la denominada Nueva Cultura del Agua, que no preconiza otra cosa que un cambio de paradigma hacia la sostenibilidad ambiental, económica, social y cultural, orientado a una consideración ecosistemática y no sólo patrimonial del agua. Es decir, se propone un cambio cultural, basándose en la consideración de los recursos hídricos como un patrimonio de la biosfera, como elemento transversal y básico para cubrir las funciones esenciales de supervivencia de todos los seres vivos. Es necesario, por tanto, una nueva forma de gestión, un cambio de las habituales estrategias de oferta al actual reconocimiento como elemento fundamental básico para satisfacer las necesidades esenciales de consumo humano, salud, higiene y producción de alimentos.

En suma, hay que alejarse del commodity económico que tanto predomina en el sector del agua como en el del control y la regulación del flujo migratorio, dejando de concebir ambos parámetros como bienes públicos de los que se sustrae un beneficio económico, para hablar desde una perspectiva humana, a través de la que se garantice la correcta regulación y protección de los Derechos Humanos de las personas, con independencia de sus circunstancias personales.

\section{EL AGUA COMO ELEMENTO DE REFLEXIÓN}

El mundo global con el que convivimos pone de manifiesto la estrecha interrelación entre los diferentes parámetros o elementos de configuración del mismo: convivimos con el cambio climático, con el agujero de la capa de ozono, con la pérdida de la biodiversidad biológica y de los recursos energéticos, con la crisis geopolítica y las dificultades de acomodar los ciclos sociales a los ciclos económicos, con las nuevas patologías asociadas a los estilos de vida y con el deterioro progresivo del entorno urbano. Todo ello ha generado 
que las exigencias por el reconocimiento y la protección de los Derechos Humanos se sitúen en primer plano de actuación gubernamental; pero, en especial, se ha intrincado en las agendas de los Estados la necesidad de situar al agua en una posición preeminente para la supervivencia humana y ecosistemática. En palabras de la OMS, el $80 \%$ de las enfermedades y $1 / 3$ de las muertes en los países en vías de desarrollo proceden de la ausencia o la contaminación del recurso hídrico, al margen de la desigualdad de género que sufren millones de mujeres y niños cuando invierten más de 200 millones de horas en recoger y transportar agua a sus hogares por no tener garantizado un derecho de acceso a este recurso, amén de la lesión que ello produce en otros derechos fundamentales en estas personas (en concreto, se estima que el $17 \%$ de los 2,3 millones de muertes en el trabajo tiene su origen en la mala calidad del agua potable, un saneamiento deficiente y la falta de higiene - Naciones Unidas, 2016: 6).

Ya desde tiempos ancestrales se ha prestado una inmensa atención al agua. Nos dice Eliade (1990: 237) que en la cosmología, en el mito, en el ritual, en la iconografía... las aguas desempeñan siempre la misma función, cualquiera que sea la estructura de los conjuntos culturales de que formen parte: preceden a todas las formas y son soporte de todo lo creado. Todas las manifestaciones ancestrales sobre la importancia del agua en la vida humana evidencian la conciencia universal del valor supremo que tiene el agua como factor para el logro de las cotas más básicas de desarrollo material y espiritual del ser humano.

Sin agua no hay vida y sin un reconocimiento adecuado y universal de la misma perdemos la posibilidad de cambio en la sociedad, de eliminar la dicotomía norte-sur y actualmente sursur también, de garantizar el bienestar social a escala mundial y, por el contrario, acrecentamos sentimientos antisociales y de radicalización y ello dentro del marco de injusticia, pobreza y desintegraciones políticas actual genera nuevos conflictos avivados por motivos étnicos, migraciones, intereses económicos internos y externos ... . (Espinosa, 2001: 92).

\subsection{La relevancia del agua como elemento de cambio en la configuración de los derechos humanos}

Como consecuencia de ello, en la última década los organismos internacionales han proclamado la necesidad de implementar un cambio de políticas entorno a los derechos del hombre. Así, el Comité de Derechos Económicos, Sociales y Culturales de Naciones Unidas, a través de la interpretación del derecho a un nivel de vida adecuado que incluya alimentación, vestido, y vivienda adecuados recogido en el artículo 11 del Pacto Internacional de Derechos Económicos, sociales y Culturales, especifica que el derecho humano al alimento y al agua potable es indispensable para vivir dignamente: el agua es un recurso natural limitado y un bien público fundamental para la vida y la salud. El derecho humano al agua es indispensable para vivir dignamente y es condición previa para la realización de otros derechos humanos (Observación General $\mathrm{n}^{\circ} 15$ ). Es decir, que el agua se configura como un elemento fundamental, básico, elemental para el desarrollo y la convivencia humana, desde el nivel biológico más esencial de la supervivencia humana hasta el desarrollo del más alto nivel de vida, pues garantiza un adecuado nivel y desarrollo de muchos otros derechos sí considerados por la comunidad internacional como humanos (vida, salud, alimentación, vivienda...), ya que salud y bienestar humano no pueden entenderse sin un adecuado acceso al agua como elemento componente del mismo y dador de vida. Así se pronunció NNUU en el Año Internacional del Agua: el derecho humano al agua es indispensable para llevar a cabo una vida en dignidad humana. Es un pre-requisito para la realización de otros derechos humanos. 
Es clara la interrelación entre los Derechos Humanos en su globalidad, siendo los mismos indivisibles, universales e interdependientes, pero para cuya efectividad, desafortunadamente, se requiere la acción de los Estados, pues la consecución de un progreso duradero en la aplicación de los Derechos Humanos depende de unas buenas y eficaces políticas nacionales e internacionales de desarrollo económico y social (Proclamación de Teherán, Naciones Unidas, 1968) y no deberíamos hacerlos depender de determinadas circunstancias o condicionantes, tales como el nivel de desarrollo de los Estados, la pobreza, el género o la propiedad, ni dejar su justificación y vinculación jurídica en manos de clasificaciones de derechos de décadas pasadas, pues todos los derechos, sean civiles y políticos, como económicos, sociales y culturales e incluso los de nueva catalogación, son componentes activos del desarrollo del bienestar último del ser humano, sin que ello sirva de excusa para hablar de derechos de segunda, cuya garantía es inexistente y cuya titularidad práctica se desplaza a los individuos o grupos más desfavorecidos.

Pero la práctica actual nos demuestra que son justamente los países en vías desarrollo y los grupos de personas en situación de desigualdad las que están implementando nuevas políticas sociales hacia el reconocimiento universal de todos los derechos del hombre, sin excepción. Véase uno de los ejemplos que se están sucediendo en los países latinoamericanos con cambios normativos estructurales, el de la Mesa por la Soberanía Alimentaria del El Salvador, la cual propone un cambio del artículo 69 de la Constitución salvadoreña para incluir el derecho humano al agua dentro del catálogo de derechos a proteger por el Estado. Al artículo actual, que ya reconoce el derecho a la alimentación, se añadiría: el agua es un recurso esencial para la vida, en consecuencia, es obligación del Estado aprovechar y preservar los recursos hídricos y procurar su acceso a los habitantes. Con esta acción, se permite cumplir con las exigencias internacionales y garantizar el bienestar y la paz social de los ciudadanos. Por tanto, reconocer que el acceso a una alimentación adecuada, al agua y al saneamiento son Derechos Humanos que los Estados deben reconocer, proteger y respetar a todas las personas es la única vía para lograr la seguridad alimentaria e hídrica en el Planeta (Ongawa, 2012: 8). Es de suma importancia situar el reconocimiento humano del derecho al agua en primera fila, pues es un elemento tan vital, transversal e interrelacionado con el resto de derechos que dan esencia a los hombres y a las sociedades en las que habitan, que el Informe sobre Desarrollo Humano de 2006 afirmaba que la falta de agua puede representar la diferencia entre salud y enfermedad, entre vida y muerte (1.200 millones de personas viven sin un acceso al agua potable, 2.600 millones no disponen de servicios mínimos de saneamiento y 2.400 millones de personas carecen de condiciones sanitarias dignas - PNUD, 2006). De ahí que en 2010 la Asamblea General de Naciones Unidas lo reconociera como un Derecho Humano, a pesar de no ser un texto vinculante jurídicamente para los Estados, pero sí una respuesta social a las actuales demandas al más alto nivel.

Esta solución intermedia, en lugar de satisfacernos nos debería servir para reflexionar sobre por qué no somos capaces de tener un sistema vinculante por igual para todos, si regulaciones sectoriales, como el Derecho Internacional Humanitario, desde sus orígenes ha sido capaz de destacar la importancia que para las personas tiene el recurso hídrico, quedando prohibido, en tiempos de guerra, obstaculizar el acceso al mismo. Por tanto, el agua nos debe servir como punto de inflexión y reflexión para producir un sistema de garantías sobre los derechos del hombre cuyo carácter universal se predique para todos los individuos sin excepción y quede garantizado para grupos de personas desfavorecidas y para situaciones peliagudas a las que los Estados no hacen frente, como es la adecuada regulación de la migración internacional. 


\section{A MODO DE CONCLUSIÓN}

La relevancia actual de los Derechos Humanos y su papel central en la filosofía práctica vendría dada por su configuración como línea transversal que cruza las dimensiones de lo ético, lo jurídico y lo político, dado que poseen un sustrato que los configura como aspiraciones morales comunes a la Humanidad entera, conformando así el único código mínimo de un ética universalmente aceptada (Zaragoza, 2015: 31). La configuración de los Derechos Humanos ha sido el resultado de una constante lucha a lo largo de la evolución histórica, una lucha que sin duda reivindican las clases sociales más desfavorecidas y gracias a las cuales nos encontramos con nuevas regulaciones normativas que permiten llevar a cabo acciones por parte de los Estados y que, en ocasiones, nos recuerdan que los elementos de vida más básicos son los que requieren de mayor regulación y protección.

Ello es lo que ocurre con el Derecho Humano al agua, ausente en la normativa internacional de clasificación de derechos por un simple acto de olvido, pues se consideraba tan básico y necesario que no se estimó necesaria su regulación. Pero la actual situación de crisis geopolítica, social, ambiental y de recursos lo ha situado a la cabeza de la lucha por alcanzar un desarrollo de bienestar máximo en el que podamos vivir dignamente, dotado de carácter universal y equitativo para todo ser humano sin distinción alguna. Especialmente notable en los sectores, países o grupos de personas desprovistas de garantías estatales como son los migrantes.

Las migraciones son consustanciales a la Humanidad, son un fenómeno estructural que hoy hunde sus raíces en las profundas desigualdades del Planeta (APDHA, 2014: 57), por lo que hay que actuar para revertir dichas situaciones entorno a un enfoque macrocooperativo, sustentado en los principios básicos de los Derechos Humanos: no discriminación, inclusión de los grupos vulnerables, participación, empoderamiento, transparencia y rendición de cuentas, de forma que Derechos Humanos y desarrollo vayan de la mano. Ante la acuciante crisis de gobernabilidad es necesario un cambio de paradigma hacia políticas que sitúen en su centro de acción a las personas como seres humanos y no como commodities, titulares de derechos universales, básicos y necesarios para el desarrollo de su personalidad, alcanzando con ello el bienestar vital.

Como dice Levenzon (2009: 47), el enfoque de Derechos Humanos exige que las políticas y las leyes no se centren únicamente en la ampliación de coberturas, sino que se debe dar prioridad a las personas más vulnerables, pues el derecho internacional dispone que toda persona, sin discriminación, debe tener acceso a los Derechos Humanos Fundamentales. Ahí es donde encontramos el punto de conexión o simbiosis entre el agua, elemento básico para el desarrollo personal, y la necesidad de universalizar su regulación para que no pueda ser utilizado como medio de desigualdad y exclusión social, especialmente notorio en determinados sectores de la sociedad, como lo son las mujeres y los niños que sacrifican su desarrollo personal, su derecho a la salud, su derecho a la educación... por conseguir un mínimo de agua para su comunidad o las personas migrantes, forzadas por la escasez de recursos y de medios de vida que huyen de sus orígenes hacia destinos más proclives en bienestar.

En la actualidad, hay alrededor de 232 millones de migrantes, pero sin embargo son en gran medida invisibles y la mayoría de ellos tienden a vivir y trabajar en la penumbra, privados de derechos y de libertades y desproporcionadamente vulnerables a la discriminación y a la marginación (Naciones Unidas, 2014: 1), empujados a vagar de un país a otro en busca de mejores condiciones de vida y de trabajo, pero también auspiciado por las grandes desigualdades entre países e incluso dentro de un mismo país y acrecentado todo 
ello, en la actualidad, por las consecuencias medioambientales de un palpable cambio climático que acaba con los recursos naturales de los que muchos migrantes vivían en sus países de origen.

Por ello es tan necesario garantizar la adecuada regulación y protección de todos los Derechos Humanos sin excepción, empezando por el más básico e hilo conductor de los demás, como es el reconocer un acceso universal al agua y al saneamiento, paliando de esta manera las deficiencias en materia de alimentación y salud de la población desfavorecida e instaurando un sistema de garantía que permita acabar con situaciones como las mencionadas. De ahí que situemos al agua como punto de reflexión para la implementación de nuevos paradigmas sociales, porque si comprendemos la importancia de reconocer su carácter humano y universal estaremos también reconociendo al mismo tiempo un trato igual y equitativo para todo ser humano, con independencia de su origen, status o situación legal en un país.

\section{BIBLIOGRAFÍA}

Asociación Pro Derechos Humanos de Andalucía-APDHA- (2014) Derechos Humanos en la Frontera Sur (consulta 20/03/2017), https://goo.gl/KKRvEs

Auxín, T. (2013) "Bienes públicos que dan la vida: el derecho humano al agua y al alimento", CSICEsfera del Agua (consulta 27/03/2017), https://goo.gl/1AbF2c

Comisión Interamericana de Derechos humanos (2013) "Los migrantes son seres humanos con derechos humanos", Comunicado de prensa de 18 de diciembre. Ginebra/Washington D.C. (consulta 27/03/20147), https://goo.gl/mGUSvv

Cornelio Landero, R. (2015) "Los derechos humanos de los inmigrantes de la frontera sur de México", en Barataria. Revista Castellano-Manchega de Ciencias Sociales, 19: 139-150, DOI: http://dx.doi.org/10.20932/barataria.v0i19

Cortizo Álvarez, J. (1993) "Tipología de las migraciones internacionales”, en Polígonos: Revista de Geografia, 3: 9-24, DOI: http://dx.doi.org/10.18002/pol.v0i3

Domenech, E. (2013) "Las Migraciones son como el agua: hacia la instauración de políticas de control con rostro humano. La gobernabilidad migratoria en Argentina", Revista Polis, 35, https://polis.revues.org/9280

Eliade, M. (1990), Tratado de historia de las religiones. Barcelona: Círculo de Lectores.

Espinosa, L. (2011) "Reflexiones sobre el agua: un espejo de nuestro tiempo", Dilemata, 6:81-99, https://goo.gl/F5keRP

Ghosh, B. (2008) "Derechos Humanos y migración: el eslabón perdido", Migración y Desarrollo, 10: 36-63, https://goo.gl//zw6MW

Levenzon. F. (2009) "Implementación efectiva el derecho al agua en América Latina”, Conclusiones del 4 Foro Agua para el desarrollo-El derecho humano al agua. Madrid: Fundación C. Isabel II.

Loperena Rota, D. (1999) "Los derechos al medio ambiente adecuado y su protección", Medio Ambiente\&Derecho. Revista Electrónica de Derecho Ambiental, 3, (consulta a 10/02/2017), https://goo.gl/hcUlZj

Mezzadra, S. (2012) "Capitalismo, migraciones y luchas sociales. La mirada de la autonomía", Nueva Sociedad, 237, https://goo.gl/HYtdA0

Naciones Unidas (2014) Los Derechos Económicos, sociales y culturales de los migrantes en situación irregular, Nueva York y Ginebra, (consulta 15/12/2016), https://goo.gl/RJw1uu

Naciones Unidas (2016) Informe de Naciones Unidas sobre el Desarrollo de los Recursos Hídricos en el Mundo: Agua y empleo. París: UNESCO, (consulta 20/12/2016), https://goo.gl/DCDZHc

Ongawa (2012) "Agua y alimentación por derecho", Derecho Humano al Agua, Vol. 6, (consulta 17/03/2016), https://goo.gl/ROZ4n5 
Organización Mundial de la Salud y Unicef (2014) Programa conjunto de Monitoreo de agua potable y saneamiento, Informe de Actualización. Nueva York (consulta 20/10/2016), http://www.wssinfo.org/

PNUD (2006) Más allá de la escasez: poder, pobreza y crisis mundial del Agua, Informe sobre Desarrollo Humano, (consulta 13/09/2016), https://goo.gl/byS21I

Red-Desc (2016) "La intersección entre el acceso a la Tierra y los derechos económicos, sociales y culturales de las mujeres", Documentos Informativos del Grupo de Trabajo sobre Mujeres y DESC, Ginebra, (consulta 10/03/2017), https://goo.gl/3EYJqI

Zaragoza Martí, M.F. (2015) La tutela multilevel del derecho al agua (tesis doctoral). Elche-Alicante: Universidad Miguel Hernández.

\section{Breve currículo:}

\section{María Francisca Zaragoza Martí}

Profesora de derecho constitucional de la Facultad de Derecho de la Universidad de Alicante (España). Doctora en Derecho Mención Internacional por la Universidad Miguel Hernández de Elche (UMHEspaña). Licenciada también en Ciencias Políticas y de la Administración (CCPP). Máster en Diplomacia y Relaciones Internacionales. Ha trabajado en la Secretaría de Estado para la Unión Europea y en la Oficina Europe Direct (España). Ha impartido clases de derecho constitucional e instituciones básicas de derecho público en la UMH en la Licenciatura de Derecho y en CC.PP. y en la Diplomatura de Relaciones Laborales, así como en los prácticums de Derecho. 